\title{
Rheumatoid arthritis pharmacogenomics
}

Rheumatoid arthritis is a highly heterogeneous disease in all its aspects, including response to therapy. During the last 10 years, we have seen evidence of the revolution of biological therapies in the treatment of rheumatoid arthritis. However, there is a substantial proportion of patients who do not respond efficiently to some of these therapies. One of the main aims for pharmacogenomics of rheumatoid arthritis for the next few years will be to accurately identify, from available therapies, which is the optimal therapy for any particular individual. The use of more powerful technologies together with the existence of large collections of samples and high-quality associated clinical data will be essential to reach this objective.

\section{KEYWORDS: anti-TNF- $\alpha$ therapy genomics methotrexate microarrays response predictor rheumatoid arthritis}

\section{Past}

Rheumatoid arthritis (RA) is a chronic inflammatory disease of the joints and one of the most prevalent autoimmune diseases in the world. RA is characterized by a persistent inflammation in the synovial joints, which ultimately leads to pain, joint erosion and functional impairment. RA is a highly heterogeneous disease, and therefore it can be difficult to diagnose in its early manifestations. Currently, RA is diagnosed following a set of diagnostic criteria established in 1987 by the American College of Rheumatology (GA, USA) [1].

Since the first clinical trials in the 1980 s up until today, the folate analog methotrexate (MTX) has been used as the first-line disease modifying antirheumatic drug (DMARD). Initially used as a chemotherapeutic in leukemia, MTX has been shown to be beneficial in reducing the level of inflammation in RA, although the precise mechanism of its action is still unknown [2]. Multiple other DMARDs have been developed, such as azathioprine, leflunomide, chloroquine, sulfasalazine or ciclosporin $A$ [3]. Although this is a relatively large arsenal of drugs, they all have the existence of substantial toxicity and relatively low efficacy in common. Thus, before 2000, remission in RA was hardly thought to be a possibility. The clinical specialist had to empirically determine the effectiveness of a particular DMARD on each patient and, in many cases, the disease progressed to more severe forms.

\section{Present}

The completion of the human genome sequence in 2001 is a landmark in human history. It is the fundamental basis from which more complex objectives, like the genomic characterization of the response to treatment, can be pursued. Not surprisingly, as soon as the sequences of the genes coding for the enzymes metabolizing DMARDs were characterized, they were studied in relation to the differential response to this treatment in RA. For example, in these last 10 years, more than 50 different studies have analyzed the relation between SNPs and the efficacy or toxicity of MTX in RA [4]. After this intense research it is now clear that the effects that genetic variations in metabolizing enzymes have on the response to DMARDs are smaller than initially expected. Although there is considerable evidence in favor of an association between some of these variants and treatment response, it is clear that higher sample sizes will be needed to precisely determine their penetrance. The lack of consistent replicability has also revealed the need for standardized approaches to characterize safety and toxicity in RA. If the terms by which we measure the effect of a compound are not very similar, comparison between the different studies will be severely weakened. Also, if we do not use more homogeneous cohorts, we risk including confounders that will reduce the power of our pharmacogenomic studies [5].

The turn of the century has seen a fundamental advance in the treatment of RA. Studies during the 1990s confirmed the ability of TNF- $\alpha$

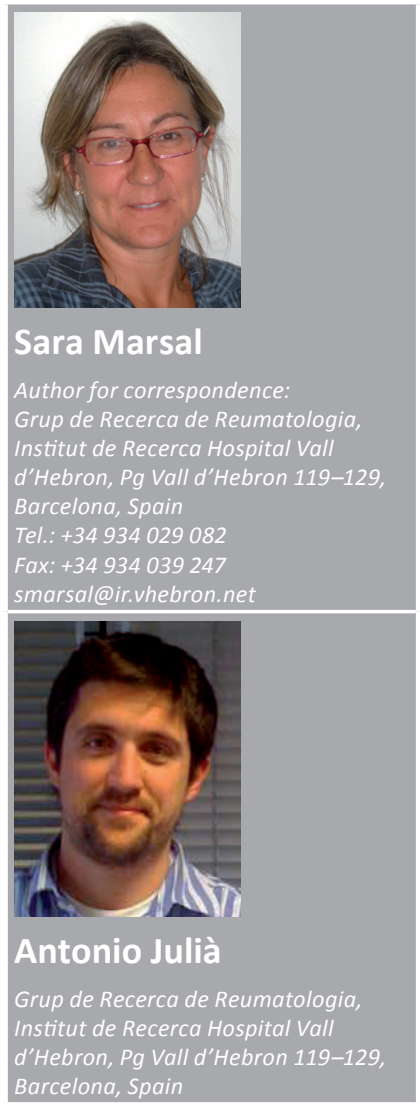


neutralizing agents to tightly control the progression of the disease. TNF- $\alpha$ is a powerful proinflammatory cytokine that is overexpressed in the synovial membrane of RA patients. The blocking of TNF- $\alpha$ either by monoclonal antibodies (infliximab and adalimumab) or by decoy soluble receptors (etanercept), has shown to be a powerful means to reduce inflammation and avoid the development of erosions. Importantly, anti-TNF- $\alpha$ treatments have introduced the possibility of remission for RA patients [6]. RA is, however, a highly heterogeneous disease and it was soon realized that there is a substantial fraction of patients $(20-40 \%$ depending on the study) that do not respond to these treatments. Together with the insufficient data on longterm safety concerns and the high costs of these therapies, there started to be a growing need to identify predictors of response to treatment with anti-TNF- $\alpha$ agents.

One of the first approaches to finding genetic predictors of response to anti-TNF- $\alpha$ therapy in RA came from the study of the polymorphisms of the gene encoding the cytokine itself. Although some studies report a positive association of $T N F-\alpha$ promoter polymorphisms with treatment response, which are known to influence the levels of expressed TNF- $\alpha$, there are also studies showing a lack of influence on the response. Again, methodological issues such as the time at which the response is measured and the system used to measure this response could explain the existence of discrepancies. With the current information it is clear, however, that the information on TNF- $\alpha$ gene variation is insufficient to carry out any prediction in the clinical setting. Similarly, there are discrepancies with other candidate genes such as HLA-DR, FCGR3 or ILIRN [7,8].

During recent decades advances in the ability to obtain biological data from samples have been almost exponential. One of the most important technological achievements was the introduction at the beginning of the 21st Century of gene-expression microarrays. Before this highthroughput technology it was only possible to study the expression of a small number of genes at a time. With microarrays it is now possible to measure the full transcriptional activity of a particular cell type or tissue. This is a powerful approach since, for the first time, the researcher does not need to be bound by the current knowledge of the pathophysiology of the disease and, instead, can study the biological system as a whole. This power was soon exploited in the characterization of the molecular mechanisms of
RA [9] and, more recently, in the identification of RNA profiles predictive of the response to antiTNF therapies [10,11]. Much like the single-gene association studies, however, the variability in the robustness of methodological approaches and the number of patients studied should be taken into account before considering the significance of the results. As with any predictor system, they will need to be tested under different clinical scenarios to confirm their utility.

\section{Future}

Following anti-TNF- $\alpha$ treatment success, intensive pharmacological research has led to the identification of new therapies for RA with very good prospects in terms of efficacy and safety. For example in 2006, an anti-CD20 antibody (rituximab) was approved for the treatment of RA. At present, other treatments have also been approved for the treatment of RA such as anti-IL-6 (tocilizumab) or anti-CTLA4 (abatacept) therapies, and many others will surely be approved in the coming years. With each new therapy that appears, the probability for any given patient to find a highly efficient therapy rises. Thus, the most ambitious objective in RA treatment, the complete remission of the disease, is becoming a reality. More than ever, we will need systems that efficiently predict which is the best therapy for each patient.

The characterization of genomic biomarkers associated with the response to biologic therapies in RA is advancing at a fast pace. Like antiTNF- $\alpha$ response predictors, new sets of genomic markers will be identified that can predict the responses to the growing panel of available treatments [12]. Also, with time, an increasing percentage of patients will reach remission and, with this, it will be necessary to identify biomarkers for therapy withdrawal. Once we have reached minimal residual activity in RA, we should be able to identify those patients who no longer need biological therapy from those who are at risk of relapse.

What will be the steps necessary to introduce genetic and genomic information into day-today clinical practice of RA? First of all we need to find robust and reproducible biomarker patterns. Technically, we are improving at a very high speed with more powerful high-throughput technologies appearing periodically. However, it will be necessary to implement more costefficient technologies if we want to facilitate the introduction of genomics into clinical practice. Also, the analytical potential of these technologies is limited by two fundamental factors, 
the quality of the biological samples and the completeness of the clinical data associated with them. Moreover, the number of available samples for each study will be crucial. For all these reasons, large collections of standardized samples with comprehensive clinical data, such as population- and disease-specific biobanks, will be likely to increase in the next few years [13].

Finally, another fundamental aspect for the introduction of pharmacogenomics in RA management, and for any complex disease in general, will be the engagement of the medical community with this new type of medical information [14]. Clinical practitioners will need to be educated and trained from their undergraduate medical studies in the use (and misuse) of genomic information for the treatment of patients. This will not be an immediate process but it is going to become a necessity in the practice of future medicine.
The introduction of new genetic and molecular markers for the determination of the optimal treatment strategy will be a reality in RA and many other common diseases in the near future. The quality of life of a patient diagnosed with RA before 2000 from one diagnosed now has changed drastically. It is our mission to maintain this pace of improvement for the next 10 years.

\section{Financial \& competing interests disclosure}

The authors have no relevant affiliations or financial involvement with any organization or entity with a financial interest in or financial conflict with the subject matter or materials discussed in the manuscript. This includes employment, consultancies, honoraria, stock ownership or options, expert testimony, grants or patents received or pending, or royalties.

No writing assistance was utilized in the production of this manuscript.

\section{Bibliography}

1 Arnett FC, Edworthy SM, Bloch DA et al.: The American Rheumatism Association 1987 revised criteria for the classification of rheumatoid arthritis. Arthritis Rheum. 31(3), 315-324 (1988)

2 Ranganathan P: An update on methotrexate pharmacogenetics in rheumatoid arthritis. Pharmacogenomics 9(4), 439-451 (2008).

3 O'Dell JR: Therapeutic strategies for rheumatoid arthritis. N. Engl. J. Med. 350(25), 2591-2602 (2004).

4 Fisher MC, Cronstein BN: Metaanalysis of methylenetetrahydrofolate reductase (MTHFR) polymorphisms affecting methotrexate toxicity. J. Rheumatol. 36(3), 539-545 (2009).

5 Plenge RM, Criswell LA: Genetic variants that predict response to anti-tumor necrosis factor therapy in rheumatoid arthritis: current challenges and future directions. Curr. Opin. Rheumatol. 20(2), 145-152 (2008).
6 Klareskog L, Catrina AI, Paget S: Rheumatoid arthritis. Lancet 373(9664), 659-672 (2009).

7 Coenen MJ, Toonen EJ, Scheffer H et al.: Pharmacogenetics of anti-TNF treatment in patients with rheumatoid arthritis. Pharmacogenomics 8(7), 761-773 (2007).

8 Bansard C, Lequerré T, Daveau M et al:: Can rheumatoid arthritis responsiveness to methotrexate and biologics be predicted? Rheumatology (Oxford) 48(9), 1021-1028 (2009).

9 van der Pouw Kraan TC, van Gaalen FA, Huizinga TW, Pieterman E, Breedveld FC, Verweij CL: Discovery of distinctive gene expression profiles in rheumatoid synovium using cDNA microarray technology: evidence for the existence of multiple pathways of tissue destruction and repair. Genes Immun. 4(3), 187-196 (2003).

10 Lequerré T, Gauthier-Jauneau AC, Bansard C: Gene profiling in white blood cells predicts infliximab responsiveness in rheumatoid arthritis. Arthritis Res. Ther. 8(4), R105 (2006).
11 Julià A, Erra A, Palacio C et al.: An eight-gene blood expression profile predicts the response to infliximab in rheumatoid arthritis. PLoS One 4(10), E7556 (2009).

12 Julià A, Barceló M, Erra A, Palacio C, Marsal S: Identification of candidate genes for rituximab response in rheumatoid arthritis patients by microarray expression profiling in blood cells. Pharmacogenomics 10(10), 1697-1708 (2009).

13 Gurwitz D, Fortier I, Lunshof JE, Knoppers BM: Research ethics. Children and population biobanks. Science 325(5942), 818-819 (2009).

14 Frueh FW, Gurwitz D: From pharmacogenetics to personalized medicine: a vital need for educating health professionals and the community. Pharmacogenomics 5(5), 571-579 (2004). 